\title{
Filosofía, crítica y práctica de sí: entre Wittgenstein y Foucault
}

\author{
Natalia Lorio* y Andrea Torrano ${ }^{* *}$
}

Resumen: Si bien Wittgenstein y Foucault pertenecen a distintas tradiciones Wittgenstein a la filosofía analítica y Foucault a la filosofía continental- es posible señalar cierta problemática común sobre lo que comprendieron como actividad filosófica. En este artículo nos ocuparemos de la actividad filosófica a partir de las nociones de crítica y práctica de sí. Tanto Wittgenstein como Foucault realizan una crítica al modo tradicional como se ha comprendido la filosofía, como también al modo de hacer filosofía. Asimismo, la tarea filosófica supone una práctica de sí, la cual puede ser considerada como propedéutica -Wittgenstein- o como parrhesía-Foucault.

Palabras clave: Filosofía, crítica, práctica de sí, Wittgenstein, Foucault

Abstract: Although Wittgenstein and Foucault belong to different traditions Wittgenstein belongs to Analytic philosophy, and Foucault to Continental philosophyit is possible to indicate some common problems about what they understood as philosophical activity. In this article we will focus on the philosophical activity from the notions of critique and practice of the self. Both Wittgenstein and Foucault carry out a critique of the traditional way of understanding philosophy and the way of doing philosophy. Likewise, the philosophical task involves a practice of the self, which can be considered like propaedeutic -Wittgenstein- or as parrhêsia-Foucault.

Keywords: Philosophy, critique, practice of the self, Wittgenstein, Foucault

\footnotetext{
* Profesora y Licenciada en Filosofía por la Universidad Nacional de Río Cuarto y Doctora en Filosofía por la Universidad Nacional de Córdoba. Es Becaria Posdoctoral en IDH-CONICET. Ha publicado en revistas especializadas, argentinas y del exterior, dos libros en coautoría y dos compilaciones. Forma parte del Comité editorial de la Revista Nombres. Dirección electrónica: natalialorio@yahoo.com.ar.

** Licenciada y Doctora en Filosofía y Licenciada en Comunicación Social por la Universidad Nacional de Córdoba. Se desempeña como Profesora Asistente en la Cátedra "Concepciones Filosóficas" en la Facultad de Ciencias Sociales de la Universidad Nacional de Córdoba. Es Investigadora Asistente en CIECS-CONICET. Ha compilado tres libros y ha publicado artículos en revistas especializadas, argentinas y del exterior. Dirección electrónica: andreatorrano@yahoo.com.ar
} 


\section{Introducción}

Wittgenstein y Foucault pertenecen a distintas tradiciones -Wittgenstein a la filosofía analítica y Foucault a la filosofía continental. No obstante, es posible señalar cierta problemática común, aunque abordada desde distintas perspectivas, que permite advertir un encuentro entre ambos. Para ello proponemos una lectura de estos pensadores a partir no ya de esas dos tradiciones enfrentadas, sino de la distinción propuesta por la filosofía francesa entre la "filosofía de la conciencia o del sujeto" y la "filosofía del concepto", que a nuestro entender permite analizar de una manera transversal las propuestas filosóficas de ambos autores. ${ }^{1}$ Bajo esta distinción podemos comprender, tal como advierte Foucault, una "línea que separa la filosofía de la experiencia, el sentido y el sujeto, de una filosofía del saber, la racionalidad y el concepto." ${ }^{2}$ Es en esta última caracterización que se inscribe el propio Foucault, y allí también podemos incluir a Wittgenstein.

De acuerdo con Veyne, Foucault y Wittgenstein "tienen en común el hecho de creer sólo en singularidades, en rechazar la verdad como adaequatio mentis et rei y estar persuadidos de que algo en nosotros (el discurso o, según Wittgenstein, el lenguaje) piensa más allá en nuestro lugar de lo que nosotros mismos pensamos". 3 Para Wittgenstein, la vida se sustenta a través de los "juegos de lenguaje" en los que está inmersa, mientras que para Foucault se trata de "juegos de verdad" que responden a una norma. Según Veyne, el uso de la expresión "juego de verdad” por parte de Foucault responde a la intención

\footnotetext{
${ }^{1}$ Karczmarczyk, propone una lectura de la filosofía de Wittgenstein a partir de esta distinción propuesta por Jean Cavaillès entre "filosofía del concepto" y "filosofía de la conciencia o del sujeto", donde el pensador austríaco se inscribe en la denominada filosofía del concepto. Tal caracterización permite establecer una aproximación con el pensamiento de Foucault, quedando pendiente la exploración de la relación entre Wittgenstein y Althusser, lo cual es desarrollado en dicho artículo. Karczmarczyk, Pedro, "Wittgenstein, la filosofía del concepto y la estrategia de su Filosofía", Tópicos. Revista de filosofía de Santa Fe, No 33, junio 2017, pp. 77-111.

${ }^{2}$ Foucault, Michel, "La vida: la experiencia y la ciencia", en Giorgi, Gabriel y Rodríguez, Fermín (comps.), Ensayos sobre biopolítica. Excesos de vida, traducción de F. Rodríguez, Buenos Aires, Paidós, 2009, p. 42.

3 Veyne, Paul, Foucault. Pensamiento y vida, traducción de María José Furió Sancho Buenos Aires, Paidós, 2014, p. 64.
} 
de diferenciarse de Wittgenstein. Pero si atendemos a lo que Foucault define por "juegos de verdad" como "conjunto de reglas de producción de la verdad" a lo que agrega que "en un juego de verdad dado, siempre cabe la posibilidad de descubrir algo diferente y de cambiar más o menos tal o cual regla", 4 es imposible no advertir una cercanía entre ambos pensadores.

Más allá de las referencias (generalmente implícitas) de Foucault acerca de Wittgenstein, nos interesa señalar que si bien la tradición de la filosofía del concepto fue la que más se dedicó a las tareas especulativas, fue también la que tomó partido durante la guerra: "como si el problema del fundamento de la racionalidad no pudiera disociarse de la interrogación sobre las condiciones actuales de existencia." 5 Esto nos conduce a afirmar que tanto la filosofía de Foucault como la Wittgenstein han tenido profundas implicancias en el pensamiento político y social. Es bajo esta mirada que nos preguntamos por la estrecha conexión que la filosofía (y el/la filósofo/a) se liga a la crítica, a la práctica de sí y a la forma de vida.

En las últimas décadas ha habido una producción en torno al vínculo entre el pensamiento de Wittgenstein y de Foucault, en este contexto puede mencionarse como pionero el artículo de Harry Aron, "Wittgenstein's Impact on Foucault" (1978), donde advierte una profunda afinidad entre ambos autores en relación con el silencio/los límites que cada uno impone sobre el lenguaje/discurso ${ }^{6}$ y de Steven Shaviro "From Language to 'Forms of Life': Theory and Practice in Wittgenstein" (1986), quien al final del artículo establece un paralelismo entre la concepción wittgensteniana de los juegos de lenguaje como prácticas sociales e históricas y la analítica del poder y de las prácticas discursivas analizadas por Foucault. Asimismo, podemos mencionar los trabajos de Ian Hacking (2002) y de Arnold Davidson (2004), quienes

\footnotetext{
${ }^{4}$ Foucault, Michel, "La ética del cuidado de sí como práctica de la libertad", en Obras esenciales, traducción de Fernando Álvarez-Uría y Julia Varela, Buenos Aires, Paidós, 2010, p. 1042.

${ }^{5}$ Foucault, Michel, "La vida: la experiencia y la ciencia", en Obras esenciales, ed. cit., p. 43.

${ }^{6}$ Este artículo fue una conferencia brindada por Aron en el marco del Segundo Simposio Internacional sobre Wittgenstein realizado entre el 29 de agosto y el 4 de septiembre de 1977 en Kirchberg, Austria, cuya temática fue Wittgenstein y su impacto en el Pensamiento Contemporáneo, cuando Foucault estaba con vida.
} 
advirtieron cómo ciertos conceptos de ambos autores pueden ser utilizados para un análisis práctico de cuestiones históricas y epistemológicas. Más recientemente, como resultado de un encuentro realizado por investigadores/as de la Escuela Normal Superior de París en el 2007, se publica Foucault, Wittgenstein: de possibles rencontres (2011), editado por Arnold Davidson y Frédéric Gros, donde se aborda la crítica de ambos autores a las teorías filosóficas tradicionales, especialmente en relación con las concepciones del lenguaje representacional, la cercanía entre concepciones de práctica discursiva de Foucault y la concepción del lenguaje de Wittgenstein y la consideración en torno al sujeto, que deja de ser considerado como una sustancia eterna y externa que preexiste a las acciones, y pasa a ser constituido por las normas del lenguaje y del poder. El año pasado se publicó la compilación a cargo de Pascale Gillot y Daniele Lorenzini, Foucault/Wittgenstein. Subjectivité, politique, éthique (2016), como resultado del coloquio internacional organizado por la Universidad Paris 1 Panthéon-Sorbonne en el 2013, que pone en el centro de la escena la subjetividad, a partir del rechazo al psicologismo y la puesta en cuestión de una filosofía de la conciencia.

Además, se pueden considerar algunos artículos diseminados que pretenden establecer una relación entre Foucault y Wittgenstein para explicar el proceso de producción de conocimiento sin el recurso a un sujeto del conocimiento, de la representación y del lenguaje; ${ }^{7}$ respecto del idealismo, escepticismo y lenguaje para considerar los límites y posibilidades del constructivismo social; ${ }^{8}$ sobre la relevancia política del pensamiento de estos autores para cuestionar el totalitarismo; ${ }^{9}$ en relación con la producción de

\footnotetext{
${ }^{7}$ Ver Rivera, Silvia, "Epistemología sin sujeto cognoscente. Superación, disolución o sujeción de la subjetividad en Popper, Wittgenstein y Foucault", Estudios de Epistemología, $\mathrm{N}^{\circ} \mathrm{X}$, junio 2013, pp. 82-101.

8 Ver Olssen, Mark, "Wittgenstein and Foucault. The limits and Possibilities of Constructivism", en Peters, Michael y Stickney, Jeff (eds.), A Companion to Wittgenstein on Education, Singapore, Springer, 2017.

9 Ver Ayestarán Uriz, Ignacio, "Tecnologías del apartheid: Wittgenstein, Foucault, Negri”, Revista de filosofia, No 11, 1995, pp. 47-58.
} 
subjetividad ligada a regímenes discursivos o reglas de lenguaje; ${ }^{10}$ sobre una teoría crítica del yo presente en ambos pensadores, ${ }^{11}$ etc.

Resulta interesante aquí tomar en cuenta el vínculo que puede establecerse entre ambos a partir de la figura de Pierre Hadot en torno a la filosofía como modo de vida -ethos-, que puede operar como una suerte de gozne entre Wittgenstein y Foucault. Por un lado, Hadot es mencionado en múltiples ocasiones en La hermenéutica del sujeto de Foucault, especialmente en las lecciones de febrero, vinculado al valor de la conversión en tanto vuelta del pensamiento hacia el sí mismo y la conversión a sí mismo (que señalan hacia modos del conocimiento del sujeto y hacia la transformación de modos de ser, hacia el ethos). ${ }^{12}$ Por otro lado, en una entrevista con A. Davidson, Hadot hace mención del impacto que le causó la lectura de Wittgenstein y la importancia de este autor para pensar la filosofía como trabajo de sí sobre sí mismo -en esa entrevista no menciona a Foucault, a quien conocía, pero sí a Wittgenstein como uno de los autores del siglo XX que invita a una actividad interior-, impresionado por el modo en que conecta discurso y modo de vida. ${ }^{13}$

Quisiéramos agregar que sobre ambos pensadores se realizó una periodización con respecto a su obra, en el caso de Wittgenstein se establece una periodización entre el "primer" y el "segundo" Wittgenstein, el primero representado por el Tractatus Logico-Philosophicus (1922) y el segundo por Investigaciones filosóficas (1953). En el caso de Foucault se hace una distinción de tres etapas, la primera que representa la arqueológica (1961-1969), la segunda, la genealógica (1971-1976) y, por último, la ética (1978-1984). A pesar de que estas periodizaciones responden a la identificación de ciertos cuestionamientos

\footnotetext{
${ }^{10}$ Ver Vernaglione, Paolo B., "When Foucault meets Wittgenstein”, 2013, conferencia disponible en: https://www.researchgate.net

${ }^{11}$ Marshall, James, "A Critical Theory of the Self: Wittgenstein, Nietzsche, Foucault", Studies in Philosophy and Education, Vol. 20, 2001, pp. 75-91.

${ }^{12}$ Foucault retoma los desarrollos de Pierre Hadot sobre epistrophe y metanoia como dos modos de concebir la conversión, es decir, como dos modos de la transformación y transfiguración del sujeto y su vínculo con el conocerse a sí mismo. Ver Foucault, Michel, La hermenéutica del sujeto, traducción de H. Pons, Buenos Aires, Fondo de Cultura Económica, 2014, pp. 215-216.

13 Davidson, Arnold y Worms, Frédéric (eds.), Pierre Hadot, l'enseignemet des antiques, l'enseignement des modernes, Paris, Éditions Rue D’Ulm, 2010.
} 
que son las directrices de las reflexiones de cada uno de estos autores es posible advertir un problema común que recorre sus trabajos. En el caso de Wittgenstein es la relación del lenguaje con el mundo, que será abordada desde dos ángulos diferentes, ${ }^{14}$ mientras que en el caso de Foucault la cuestión es crear una historia de los diferentes modos a través de los cuales se transforman los seres humanos en sujetos. ${ }^{15}$

Si bien podemos reconocer ciertas derivas en las investigaciones de estos pensadores, es posible advertir tanto en Wittgenstein como en Foucault, una distancia crítica de la filosofía que se pretende totalizadora y sistemática, por una actitud o ethos filosófico que responde a un modo de emprender la tarea filosófica. Sin pretender homogeneizar las reflexiones de Wittgenstein con las de Foucault, nos proponemos en este artículo establecer un diálogo entre ambos en relación con la manera cómo comprendieron la actividad filosófica a partir de dos nociones que están estrechamente relacionadas: crítica y práctica de sí. En el primer apartado nos ocuparemos de la filosofía entendiéndola como una práctica crítica. Para ello remitiremos a las críticas que tanto Wittgenstein como Foucault realizan al modo tradicional en que se ha comprendido la filosofía, como también al modo de hacer filosofía que estos pensadores proponen. En el segundo, desarrollaremos la filosofía como una práctica de sí, la cual supone para Wittgenstein una terapéutica mientras que para Foucault una parrhesía.

\section{La filosofía como práctica crítica}

En uno de sus últimos textos, escrito bajo el seudónimo de Maurice Florence, Foucault inscribe su trabajo en la "tradición crítica de Kant" y a su proyecto lo denomina "una bistoria crítica del pensamiento". ${ }^{16}$ Foucault recupera al

\footnotetext{
${ }^{14}$ Ver O’Connor, Dan J. (comp.), Historia crítica de la filosofía occidental. VII. La filosofía contemporánea, traducción de N. Míguez, A. Pirk y N. Roble, Barcelona-Buenos Aires, Paidós, 1983, pp. 275-277.

${ }^{15}$ Ver Foucault, Michel, “El Sujeto y el Poder”, en Dreyfus, Hubert y Rabinow, Paul, Michel Foucault: Más allá del estructuralismo y la hermenéutica, traducción de R. Paredes, Buenos Aires, Nueva Visión, 2001, p. 241.

16 Foucault, Michel, "Foucault" (Ensayo autobiográfico para el Dictionaire des philosophes), en Obras esenciales, ed. cit., pp. 999-1003.
} 
Kant del "Was ist Aufklärung?" (1784). Reconoce en este escrito una interrogación por el presente, por la actualidad: "¿qué pasa hoy? ¿Qué pasa ahora?", y advierte en él un intento por reconocer un elemento singular del presente, mostrar en qué aspecto ese "ahora" es expresión de un proceso que concierne al pensamiento y, por último, señalar en qué aspecto y cómo el individuo que habla, en cuanto pensador, forma parte del mismo. ${ }^{17}$ En definitiva, se trata de interrogar sobre aquello del presente que debe ser objeto de la reflexión filosófica, sobre el acontecimiento y el carácter que la actualidad le imprime al filósofo/a. De acuerdo con Foucault,

La práctica filosófica o, más bien, el filósofo, al emitir su discurso filosófico, no puede evitar plantear la cuestión de su pertenencia a ese presente. Es decir que ya no será simplemente, o ya no será en absoluto la cuestión de su pertenencia a una doctrina o una tradición la que va a planteársele (...), sino la cuestión de su pertenencia a un presente o, si se quiere, su pertenencia a un cierto "nosotros" (...). Ese "nosotros" debe llegar a ser para el filósofo, o está llegando a ser, el objeto de su propia reflexión (...). La filosofía como superficie de aparición de una actualidad, la filosofía como interrogación sobre el sentido filosófico de la actualidad a la cual pertenece el filósofo, la filosofía como interrogación, por parte de ese "nosotros" del que él forma parte y con respecto al cual tiene que situarse. ${ }^{18}$

De este modo se establece una conexión entre filosofía, discurso filosófico y el/la filósofo/a. La filosofía es una práctica que supone una actitud, un ethos filosófico: "que se podría caracterizar como crítica permanente de nuestro ser histórico". ${ }^{19}$ Este ethos filosófico puede ser caracterizado como: a) una actitud límite, esto es, "en lo que nos es dado como universal, necesario, obligatorio, cuál es la parte de lo que es singular, contingente y debido a

${ }^{17}$ Foucault, Michel, El gobierno de síy de los otros, traducción de H. Pons, Buenos Aires, Fondo de Cultura Económica, 2009, pp. 28-29. Son varios los textos en los cuales Foucault hace referencia a este escrito de Kant; por ejemplo, "La vida: La experiencia y la ciencia" (1985), la conferencia "¿Qué es la crítica?” (1978), "El Sujeto y el poder" (1982), “¿Qué es la ilustración?” (1984).

${ }^{18}$ Foucault, M., El gobierno de si y de los otros, ed. cit., pp. 30-31.

${ }^{19}$ Foucault, Michel, “¿Qué es la ilustración?” (1984), en ¿Qué es la ilustración?, traducción de S. Mattoni, Córdoba, Alción Editora, 2002, p. 97. 
coacciones arbitrarias" 20 y b) una "actitud experimental", "un trabajo de nosotros mismos sobre nosotros mismos en tanto seres libres". ${ }^{21}$ Foucault llama a este ethos filosófico una "ontología de nosotros mismos", 22 se trata de una actitud crítica sobre lo que somos, sobre nuestros límites, en definitiva, de una prueba histórico-práctica sobre cómo llegamos a ser lo que somos y de los límites que podemos franquear.

Esta consideración de la filosofía como indagación sobre el presente y como ethos filosófico la encontramos también en Wittgenstein. Como señala Badiou, Wittgenstein -entre otros- nos incita a no olvidar "que las condiciones de la filosofía, o sea las verdades que ésta atestigua, son siempre contemporáneas". 23 Esto se traduce en un modo de ser contemporáneo, que refiere, por un lado, a que reconoce que las verdades proceden de su tiempo, por otro, a que hace de su vida el teatro de sus ideas, y de sus cuerpos, el lugar de lo Absoluto. ${ }^{24} \mathrm{La}$ filosofía así comprendida (y el ethos filosófico) responde al carácter epocal del cual el/la filósofa/a es parte. La filosofía entonces se aleja de las verdades comprendidas como eternas y las sitúa en su contemporaneidad.

Para Wittgenstein la filosofía es un método de investigación, una práctica. En el Prólogo del Tractatus ${ }^{25}$ señala que el objeto de la filosofía no es producir sistemas de verdades cerrados, sino desarrollar una actividad clarificadora que tienda a separar los problemas genuinos de aquellos carentes de sentido: "Todo el significado del libro puede resumirse en cierto modo en lo siguiente: Todo aquello que puede ser dicho, puede decirse con claridad: y de lo que no se puede hablar, mejor es callarse." Posteriormente señala que,

${ }^{20}$ Ibidem, pp. 101-102.

${ }^{21}$ Ibidem, pp. 102-103.

22 Ibidem, p. 103.

23 Badiou, Alain, La antifilosofía de Wittgenstein, traducción de María del Carmen Rodríguez, Buenos Aires, Capital Intelectual, 2013, p. 7.

${ }^{24}$ Ibidem, p. 8.

${ }^{25}$ Wittgenstein, Ludwig, Tractatus Logico-Philosophicus, traducción de Isidoro Reguera y Jacobo Muñoz, Madrid, Alianza, 2012. Toda vez que se citemos este texto se hará referencia al número de la proposición, o al de la página cuando se trate del prólogo o introducción. 
El objeto de la filosofía es la aclaración lógica del pensamiento. Filosofía no es una teoría, sino una actividad. Una obra filosófica consiste esencialmente en elucidaciones. El resultado de la filosofía no son "proposiciones filosóficas", sino el esclarecerse de las proposiciones. La filosofía debe esclarecer y delimitar con precisión los pensamientos que de otro modo serían, por así decirlo, opacos y confusos (4.112).

La función de la filosofía es "aclarar", "esclarecer", "elucidar", se trata de una actividad que supone delimitar las proposiciones que conforman nuestro saber. En este sentido es que puede hablarse de un ethos filosófico en Wittgenstein y establecer una conexión con la Aufklärung, como advierte Bouveresse, en tanto aufklären, actividad que permite echar luz, clarificar. ${ }^{26}$ Por lo cual, el/la filósofo/a es quien procura establecer tales elucidaciones.

En el Tractatus se traman una serie de proposiciones que demarcan lo decible y lo indecible, y en ese mismo acto se señala qué tipo de uso del lenguaje es coherente con estos principios y, por ende, qué forma ha de cobrar la filosofía como práctica: desde Wittgenstein toda proposición sobre la totalidad del mundo está desprovista de sentido por la simple razón de que una proposición, para tener sentido, debe tener la posibilidad al menos de ser verdadera o falsa. Por cuanto la filosofía no aparece como una doctrina, la clarificación del lenguaje que le es propia es una suerte de terapéutica (de diluir "los falsos problemas"). Si esto es logrado por el Tractatus (aunque pueda ser puesto en duda), a su vez va más allá al señalar problemas filosóficos, problemas vitales sin que podamos diluirlos en la clarificación.

Tanto en el Tractatus como en Investigaciones filosóficas, Wittgenstein se propone cuestionar el discurso filosófico concebido de modo tradicional. Esto es, el discurso filosófico como sistemático y pleno de sentido. Por el contrario, "la filosofía de Wittgenstein es un sinsentido indispensable y no un sinsentido vano (...) la filosofía no es teoría, no se resuelve en un cuerpo de verdades afirmables, sino que es una actividad". ${ }^{27}$ En relación con la actividad filosófica en Investigaciones Filosóficas, Wittgenstein expresa,

${ }^{26}$ Bouveresse, Jacques, Wittgenstein y la estética, traducción de José Javier Marzal, Valencia, Universidad de Valencia, 1993,

27 O'Connor, D. (comp.), Historia crítica de la filosofía occidental. VII. La filosofía contemporánea, ed. cit., p. 266. 
El descubrimiento real es el que me hace capaz de dejar de filosofar cuando quiero.

Aquel que lleva la filosofía al descanso, de modo que ya no se fustigue más con preguntas que la ponen a ella misma en cuestión (IF 133).

Wittgenstein no sólo cuestiona el discurso filosófico tradicional sino también el modo en el que se hace filosofía. Esto ha conducido a afirmar que es un "anti-filósofo". ${ }^{28}$ En este punto también podemos encontrar una relación con Foucault; en efecto, éste se asume más como historiador que como filósofo, ya que encuentra en el discurso filosófico una pretensión de totalidad y sistematicidad. Podríamos decir que tanto Wittgenstein como Foucault se alejan de la teoría y lo que proponen son pensamientos, preguntas, problematizaciones. La escritura fragmentaria en Wittgenstein y las investigaciones de Foucault como problematizaciones conlleva esta actitud de alejamiento de cualquier teoría filosófica.

Como señala Wittgenstein en el Prólogo de Investigaciones Filosóficas:

Tras varios intentos desafortunados de ensamblar mis resultados en una totalidad semejante, me di cuenta de que eso nunca me saldría bien. Que lo mejor que yo podría escribir siempre se quedaría sólo en anotaciones filosóficas; que mis pensamientos desfallecían tan pronto como intentaba obligarlos a proseguir, contra su inclinación natural, en una sola dirección. Y esto estaba conectado, ciertamente, con la naturaleza misma de la investigación. Ella misma nos obliga a atravesar en zigzag un amplio dominio de pensamiento en todas las direcciones.

Foucault también cuestiona el papel de la teoría que no debe ser "formular la sistematicidad global que hace encajar todo", ${ }^{29}$ sino "la teoría como caja de herramientas quiere decir: Que se trata de construir no un sistema

\footnotetext{
${ }^{28}$ Ver Badiou, Alain, La antifilosofía de Wittgenstein, ed. cit., y Shaviro, Steven, "From language to forms of life. Theory and Practice in Wittgenstein", Social Text, $\mathrm{N}^{\circ} 13 / 14$, Winter - Spring, 1986, p. 218.

${ }^{29}$ Foucault, Michel, "Poderes y estrategias", Microfísica del poder, traducción de Julia Varela y Fernando Álvarez-Uría, Madrid, La Piqueta, 1991, p. 183.
} 
sino un instrumento (...). Que esta búsqueda no puede extenderse más que gradualmente, a partir de una reflexión (necesariamente histórica en alguna de sus dimensiones) sobre situaciones dadas". 30

En el segundo Wittgenstein, la tarea de la filosofía aparece en clara oposición a la teorización: la filosofía debe clarificar el lenguaje, destruyendo malentendidos lingüísticos; ya no busca la esencia escondida, sino busca lo concerniente a esas reglas prácticas y temporales que usamos cuando utilizamos determinadas palabras. "La filosofía se limita entonces a describir la exterioridad de las cosas, sus posibilidades de combinación que se presentan concretamente sin ninguna necesidad de postular misteriosos procesos secretos". 31

Estableciendo una crítica radical al modo en que se ha desarrollado la filosofía a lo largo de su historia, Wittgenstein resitúa la tarea de la filosofía: ya no busca la fundación última de las cosas, sino que se trata de resituarlas en un conjunto de relaciones de las cuales emergen y mostrar o describir su fisonomía. La herramienta principal es el análisis del lenguaje y sus usos concretos. Resituar exteriormente el modo en que aparecen las relaciones entre las cosas y las reglas prácticas que demarcan esas posibilidades, da cuenta de la posibilidad crítica de la filosofía y su potencia para hacer aparecer las preocupaciones sobre las mismas y, de modo central, tender hacia el mejoramiento de un modo de vida de aquel que la practica. Como advierte Savoia,

el método filosófico de Wittgenstein ofrece una apertura hacia aquello que tradicionalmente es considerado extra-filosófico: su filosofía no trata de los objetos eternos e inmutables, sino por la observación del lenguaje efectivo, de fenómenos espacio-temporales determinados para atrapar/recoger/dar con su especificidad. Toda la actividad destructiva y crítica de la filosofía de Wittgenstein se

\footnotetext{
30 Ibidem, p. 184.

${ }^{31}$ Savoia, Paolo, "D'un point de vue politique. Philosophie, pratiques, pouvoirs : notes sur Wittgenstein et Foucault", en Frédéric Gros y Arnold Davidson (eds.), Foucault, Wittgenstein : de possibles rencontres, Paris, Éditions Kimé, 2011, p. 162.
} 
superpone así a un deber positivo, que no puede sino aparecer como confrontando a las pretensiones de la filosofía tradicional. ${ }^{32}$

En este sentido, como señala Shaviro, la crítica en Wittgenstein no debe ser entendida en el sentido filosófico usual: "La filosofía crítica es primeramente negativa en su funcionamiento, determina los límites más allá de los cuales el pensamiento o el lenguaje no puede ir correctamente (el proyecto de Kant)". ${ }^{33}$ Pero si bien en el Tractatus Wittgenstein parece ser crítico a la manera kantiana, en Investigaciones Filosóficas arremete contra el proyecto crítico kantiano: "Una crítica de los límites del lenguaje es equivocada como una crítica de los límites del pensamiento". ${ }^{44}$ La crítica entonces no sólo debe ser concebida como límite (de allí su sentido negativo) sino que también presenta un uso afirmativo, la crítica posibilita un modo de hacer filosofía.

Esta consideración de la crítica quizá pueda comprenderse en la relación que Wittgenstein establece entre filosofía y lenguaje. Como advierte Cabanchik, habría dos tendencias en el pensamiento de Wittgenstein; por un lado, la meta de la filosofía es levantar un muro donde termina el lenguaje, y, por otro, enseñarnos cómo debemos atravesar ese muro. De un lado, el muro del lenguaje y, del otro, el lenguaje como cinta de Moebius. ${ }^{35}$ Desde nuestra lectura, la crítica puede entenderse como límite, muro -en este caso se trataría de un sentido negativo- pero, al mismo tiempo, como límite que se puede franquear, atravesar el muro -donde se trataría de una crítica en sentido positivo.

Esta misma distinción en torno a la noción de crítica podemos rastrearla en el pensamiento de Foucault, ${ }^{36}$ como vimos anteriormente la crítica puede

\footnotetext{
32 Ibidem, p. 163.

33 Shaviro, Steven, "From language to forms of life. Theory and Practice in Wittgenstein", cit., p. 221.

34 Ibidem, p. 222.

${ }^{35}$ Cabanchik, Samuel, El abandono del mundo, Buenos Aires, Grama Ediciones, 2006, p. 98.

${ }^{36}$ Para un análisis de la noción de crítica en Foucault, ver Fimiani, Mariapaola, Foucault y Kant. Crítica Clínica Ética, Buenos Aires, Herramienta, 2005; Passerin d' Entrèves, Maurice, "Crítica e Iluminismo. Sobre Michel Foucault", en Pensamiento de los confines, 5, octubre 1998, pp. 171-182; Díaz, Esther (ed.) "Un nuevo kantismo en Foucault", La ciencia y el imaginario social, Buenos Aires, Biblos, 1996; Torrano, Andrea, "Foucault y la
} 
ser comprendida como una actitud límite -que remitiría a un sentido negativopero también como actitud experimental -esto es, en sentido positivo: "Hay que considerar la ontología crítica sobre nosotros mismos (...) como una actitud, un ethos, una vía filosófica donde la crítica a lo que somos es a la vez análisis histórico de los límites que se nos plantean y prueba de su franqueamiento posible". ${ }^{37} \mathrm{El}$ reconocimiento de los límites, para Foucault, es la actitud primordial para que puedan ser franqueados. El ethos se presenta como crítica, pero también como transgresión. ${ }^{38}$

Es este alejamiento y rechazo del discurso filosófico lo que hace que la crítica se convierta en un modo de reflexión, y, en consecuencia, en una actitud filosófica. Tanto en Foucault como en Wittgenstein la noción de crítica debe ser considerada como una actitud filosófica. La crítica no sólo debe ser entendida como crítica $a$ la filosofía (y es en este sentido que podemos hablar de una anti-filosofía en Wittgenstein y en Foucault) sino como crítica filosófica, es decir, como una actitud, un ethos, que debe asumir quien se proponga como tarea la indagación filosófica.

\section{La filosofía como práctica de sí}

Tanto en Wittgenstein como en Foucault encontramos un modo de asumir la filosofía que implica la crítica: en ambos se daría cierta vuelta a la filosofía (que no debería entenderse sólo como retorno, sino como rodeo, o dar vuelta, voltear) de la fuerza viva que toca el ser mismo del sujeto. En Wittgenstein, se trata de una sabiduría que apunta al sujeto, en la que algunos

tradición crítica de Kant", en Sebastián Torres y Julia Smola (Comps.), Lecturas contemporáneas de la filosofía política clásica y moderna, Buenos Aires-Los Polvorines, Universidad de General Sarmiento, 2012, pp. 271-280 y Torrano, Andrea, "La Aufkelärung en la ontología del presente de M. Foucault", Nómadas. Revista Crítica de Ciencias Sociales y Jurídicas, 31, julio-diciembre 2011. Disponible en: http://revistas.ucm.es/index.php/NOMA/article/view/36817

${ }^{37}$ Foucault, Michel, “Qué es la Ilustración?”, ed. cit., p. 108.

38 El ethos trasgresor y la elaboración de una estética de sí están en realidad más próximos a la visión nietzscheana de una transvaloración de los valores que a la noción kantiana de mayoría de edad. Ver Passerin d'Entrèves, Maurice, "Crítica e Iluminismo. Sobre Michel Foucault", cit., p. 181. 
han señalado la transformación (acaso enmascarada) de la filosofía como un ejercicio de sí, que supone un trabajo sobre sí mismo, bajo la premisa de ir más allá de las pretensiones de la metafísica. En Foucault nos encontramos con una filosofía que se interroga por las prácticas más que por los contenidos de sus discursos de la verdad, la filosofía se manifiesta como un acto de decir veraz, una forma particular de parrhesía, la cual implica una práctica de sí.

$\mathrm{Si}$, como sostiene McDowell, se parte de que la filosofía en Wittgenstein (en el Tractatus) es una suerte de terapéutica, cabe preguntar si no se trata de una interpretación intelectualista que no toma en cuenta la causa de la búsqueda filosófica, esa a la que hace alusión Wittgenstein sobre los problemas vitales, o las preocupaciones más claramente filosóficas, y de las que termina callando. ${ }^{39}$ Nos preguntamos entonces ¿qué supone rebasar la interpretación intelectualista? ¿Cuál es la causa de la búsqueda filosófica? ¿qué involucra esa búsqueda que no sea saber? Acaso aquí esté la indicación que buscamos para seguir el rastro de una filosofía que configura un trabajo sobre sí mismo.

Nos interesa especialmente centrarnos en el Tractatus en tanto entendemos que es allí donde se trama de un modo muy patente la relación de la filosofía con el sentido y, también aquello que -allende el sentido- es denominado "mística". En este marco, entre la filosofía y la mística se juega una crítica al sujeto de la filosofía, crítica que entendemos involucra una práctica de sí.

En el Tractatus encontramos que está presente el principio de reflexividad de la razón y las reflexiones sobre la representación de la representación, desde la certeza de que este proceso no puede ser operado sino en el lenguaje: ${ }^{40}$ en la medida en que accedemos al mundo desde o en el lenguaje, hay una parte de la relación que siempre se mantiene como inaccesible. Ese fondo, centro o arché inaccesible, lejos de ser eludido por la filosofía como práctica, ha sido aquello que ha fascinado a la filosofía. Sin embargo, en tanto que, según Wittgenstein, no es posible acceder a ese fondo, las principales proposiciones del Tractatus demarcan aquello que puede ser enunciado con sentido (factible de ser

39 Volbers, Jörg, "Le sujet de la critique chez Wittgenstein et Foucault", en Frédéric Gros y Arnold Davidson (Eds.), Foucault/Wittgenstein. De possibles rencontres, ed. cit., pp. 97-113.

${ }^{40}$ Ibidem, p. 102. 
verdadero o falso) de todo aquello que es sinsentido: "5.6. Los límites de mi lenguaje significan los límites de mi mundo" y "5.6.1. La lógica llena el mundo; los límites del mundo son también sus límites (...) lo que no podemos pensar no lo podemos decir; así tampoco podemos decir lo que no podemos pensar".

Si bien la correspondencia entre lenguaje-mundo es la matriz del sentido, esto no cierra los problemas a los que alude Wittgenstein, más bien todo lo contrario. Si las ciencias demarcan el terreno de lo decible, la filosofía tiene la tarea de poder disolver los falsos problemas delimitando aquello que se puede decir con sentido (de lo que sólo se puede mostrar). Sin embargo, la teoría presente en el Tractatus es insatisfactoria, puesto que se contradice Wittgenstein lo sabe bien- porque justamente da una visión completa de nuestra relación con el mundo.

Wittgenstein se ve forzado a recurrir a la idea de la filosofía en tanto trabajo sobre sí mismo, pues la distinción entre decir y mostrar es una distinción que no puede ser más que mostrada al sujeto. ${ }^{41} \mathrm{Si}$ del sujeto, del tiempo, de la temporalidad, de la causalidad, de la totalidad del mundo nada puede decirse (6.53. El método correcto de la filosofía sería propiamente éste: no decir nada más que lo que se puede decir), la filosofía coincidiría con las ciencias o con un análisis de lo decible que demuestren que todo el terreno de la metafísica no da significado a ciertos signos - o son un sin-sentido. Sin embargo, es esto mismo lo que se encuentra rebasado en el Tractatus.

De allí que aparece la filosofía como trabajo sobre sí mismo, una práctica que requiere el coraje de haber hecho el ejercicio de clarificación y habiendo tenido éxito en esa tarea, mostrar su fracaso:

6.54. Mis proposiciones esclarecen porque quien me entiende las reconoce a final como absurdas, cuando a través de ellas -sobre ellas- ha salido fuera de ellas (tiene por así decirlo, que arrojar la escalera después de haber subido

\footnotetext{
${ }^{41}$ En el "Prólogo" al Tractatus Wittgenstein señala: "La verdad de los pensamientos aquí comunicados me parece, en cambio, intocable y definitiva. Soy, pues, de la opinión de haber solucionado definitivamente, en lo esencial, los problemas y, si no me equivoco en ello, el valor de este trabajo se cifra, en segundo lugar, en haber mostrado cuán poco se ha hecho con haber resuelto estos problemas" (Wittgenstein, Ludwig, Tractatus Logico-Philosophicus, ed. cit., p. 42).
} 
por ella). Tiene que superar estas proposiciones; entonces ve correctamente el mundo.

La práctica de la filosofía no supone solamente la (di)solución de los problemas; ${ }^{42}$ la insatisfacción y contradicción en la que cae la práctica de la filosofía en el Tractatus, indica que éste aparece como el camino propedéutico que sólo sirve a quien ha recorrido el camino y desde allí puede remitir también a ese ámbito de lo inexpresable (del que del mundo, vinculado a nuestros problemas vitales, de los que nada sabemos). La práctica de la filosofía interviene en la seguridad del sujeto, interrumpe la delimitación previa de lo que puede expresarse y pensarse, confluye en una serie de proposiciones paradigmáticas acerca de todos aquellos problemas vitales (el enigma de la vida, que el mundo sea, etc.) que las ciencias naturales no pueden resolver y que, por ende, la filosofía tendría que señalar como límite del pensamiento. $\mathrm{Y}$, sin embargo, desde la proposición 5.6.: "Los límites de mi lenguaje significan los límites de mi mundo", el Tractatus desborda sus propias fronteras hacia lo que Wittgenstein llama lo místico, aun cuando del ámbito de la ética -como de lo místico- no puede haber teoría, es inexpresable.

Wittgenstein refiere que "El sentido de mi libro es ético", y en una carta a von Fricker dice sobre el Tractatus:

Mi obra se compone de dos partes: de la que aquí aparece; y de todo aquello que no he escrito. Y precisamente esta segunda parte es la importante. Mi libro delimita por dentro lo ético, por así decirlo; y estoy convencido de que estrictamente, sólo puede delimitarse así. Creo, en una palabra, que todo aquello sobre lo que muchos hoy parlotean lo he puesto en evidencia yo en mi libro guardando silencio sobre ello. ${ }^{43}$

La filosofía se vuelve una actividad ética (más que teórica): "no es un filósofo quien tiene un saber último, sino aquel que practica una actividad del saber a partir de una posición muy precisa, pero no superior, con coraje, buscando volverse éticamente capaz de decir la verdad, buscando

${ }^{42}$ Reguera, Isidoro y Muñoz, Jacobo, "Introducción” a Wittgenstein, L., Tractatus Logico-Philosophicus, op. cit., p. 17.

${ }^{43}$ Ibidem, p. 16. 
transformarse a fin de poder vivir mejor también a través de la práctica de la filosofía". ${ }^{44}$ Es posible ver incluso en Wittgenstein la filosofía no sólo como un trabajo sobre sí mismo, sino también como una batalla -como sostiene Stanley Cavell- ${ }^{45}$ contra los encantamientos del pensamiento, incluso una batalla ética contra nuestras prácticas y contra el lenguaje que nos constituye. Esto puede ser acaso otro punto de encuentro con Foucault, con el modo en que desarrolla la práctica de la filosofía.

Desde el Tractatus, la tarea de la filosofía como práctica de sí, como esclarecimiento, no sólo debe leerse en relación con cierta continuidad kantiana, incluso humeana, o como un corte de la afilada navaja de Occam. Puede leerse también como el acceso -en su límite- al sin sentido que no se disuelve: que se trata de aquello inexpresable, hacia ese límite del que podemos tener experiencia. El movimiento de la filosofía aquí aparece como ejercicio de pensar los límites de lo expresable y a la vez señalar todo aquello que escapa a ese objetivo (no es casual que hacia el final de la obra las proposiciones devengan aforismos). Ellos señalan hacia una dirección, señalan hacia el límite que se había trazado, pero ahora hacia afuera, hacia esa totalidad de sentido del mundo de la que nada podemos saber. ¿En qué se transforma la filosofía aquí? Si en principio la filosofía aparece como una actividad de clarificación, no como una doctrina, esa suerte de terapéutica (que diluye "los falsos problemas") es superada al situar problemas filosóficos, problemas vitales que son tales sin que podamos diluirlos en la clarificación. Lo que dice el Tractatus y lo que muestra pueden ser tomados como el señalamiento de todo aquello que queda tras el cerco. La proposición 6.52. refiere: "Sentimos que aun cuando todas las posibles cuestiones científicas hayan recibido respuesta, nuestros problemas vitales todavía no se han rozado en lo más mínimo. Por supuesto que entonces ya no queda pregunta alguna; y esto es precisamente la respuesta". El sentido de la vida no puede ser dicho, en todo caso se muestra. Y el método correcto de la filosofía (6.5.3.) se muestra en su propio hacer, en su propia práctica como insatisfactoria, yendo, a la vez, más allá de sus propios propósitos.

\footnotetext{
${ }^{44}$ Savoia, Paolo, "D’un point de vue politique. Philosophie, pratiques, pouvoirs : notes sur Wittgenstein et Foucault", ed. cit., p. 162.

${ }^{45}$ Ver Cavell, Stanley, The claim of reason, Oxford, Oxford University Press, 1979.
} 
Si el Tractatus no sólo puede pensarse como una práctica crítica que involucra a la filosofía y su discurso, sino que propone a un sujeto (del ejercicio, sea quien escribe o quien lee y sigue y comprende), es posible pensar desde aquí también la forma literaria del Tractatus -como lo lee Hadot-46 en donde las proposiciones se toman por aforismos que terminan de manera tan enigmática como "7. De lo que no se puede hablar hay que callar".

La filosofía puede pensarse como ejercicio espiritual dirigido a modificar la manera de vivir y de ver el mundo: los motivos wittgensteinianos en los que está presente la insatisfacción del saber (la ciencia) respecto de los problemas vitales (de lo que poco o nada podemos saber), las proposiciones éticas, metafísicas o místicas (los límites de nuestro mundo, los límites del sujeto, o el sujeto como límite del mundo, el hecho de que el mundo sea, la muerte, el tiempo y el espacio), aquello sobre lo que las proposiciones con sentido nada pueden decir, están atravesados por un uso indicativo del lenguaje: ${ }^{47}$ el lenguaje muestra lo que no puede expresar, donde "lo que cuenta no es lo que nos dice, sino lo que nos permite vislumbrar". ${ }^{48}$ Lo central de esta práctica filosófica, de este modo de entender la filosofía como ejercicio no es lo que puede decir, sino lo que puede callar. Técnica de sí en la que el sujeto de la experiencia asume lo inexpresable como tal y hace silencio.

Retomando el vocabulario foucaultiano de La hermenéutica del sujeto, el Tractatus tiene una dimensión espiritual en tanto se ocupa del problema del acceso a la verdad y las condiciones de aquella para una transformación espiritual del sujeto. ${ }^{49}$ Sólo que el pasadizo que lleva a esta transformación es el lenguaje y sus límites. El lenguaje, en su imposibilidad o como condición de posibilidad, es también el transporte, el soporte, la red, el límite y la experiencia de "todo eso" más allá de la lógica, de todo eso que compone la obra, pero que no está escrito (o que apenas asoma hacia el final), eso que es "la segunda parte, la importante", la ética -o lo místico- como experiencia no racional-discursiva.

\footnotetext{
${ }^{46}$ Hadot, Pierre, Wittgenstein y los límites del lenguaje, traducción de M. Arranz, Valencia, Pre-textos, 2007, p. 16.

47 Ibidem, p. 46

48 Ibidem, p. 47.

${ }^{49}$ Volbers, Jörg, "Le sujet de la critique chez Wittgenstein et Foucault”, p. 103.
} 
Sabemos que tras el llamado al silencio en el Tractatus siguen para Wittgenstein años de crisis, la práctica de la filosofía cae en cierta insatisfacción y contradicción abriendo luego a los desarrollos sobre los juegos del lenguaje. ${ }^{50}$ La centralidad que cobra la noción de práctica en Investigaciones Filosóficas y Sobre la certeza, señalan la transformación del saber, entendido ahora como aprender o comprender el juego de lenguaje, la regla y la excepción en la práctica. Este viraje en la filosofía wittgensteniana da cuenta de la radicalidad de la crítica y la práctica filosófica, de la práctica de sí como transformación de sí: no es en el ámbito del discurso que se dirime el sentido, sino en la práctica discursiva que se inscribe en un juego del lenguaje: una red de proposiciones sostiene nuestro modo de ser (225), nuestra forma de vida y el modo en el que actuamos (229), pero no es inmutable, sino que cambia con el tiempo (256). ${ }^{51}$

Por su parte, para Foucault la tarea fundamental de la filosofía ha sido advertir sobre los peligros del poder:

en su vertiente crítica -y entiendo la crítica en un sentido amplio-, la filosofía es precisamente lo que vuelve a poner en cuestión todos los fenómenos de dominación (...). Esta función de la filosofía dimana, hasta cierto punto, del imperativo socrático: "Ocúpate de ti mismo", es decir, "Fúndate en libertad, mediante el dominio de ti". 52

Foucault encuentra en los griegos, en el "cuidado de sí", un modo de ser, de comportarse, que corresponde a una filosofía como práctica de sí. De acuerdo con Foucault, en la filosofía antigua el cuidado de sí mismo fue considerado como un deber y como una técnica: "designa una ocupación regulada, un trabajo con sus procedimientos y sus objetivos". ${ }^{33}$ Es importante establecer una distinción entre el "cuidado de sî", tal como es comprendido

${ }^{50}$ Ver Baum, Wilhelm, Ludwig Wittgenstein: vida y obra, traducción de J. Ibañez, Madrid, Alianza, 1988.

${ }^{51}$ Ver Wittgenstein, Ludwig, Sobre la certeza, edición bilingüe, traducción de Josep Lluís Prades y Vincent Raga, Barcelona, Gedisa, 2000.

${ }^{52}$ Foucault, Michel, "La ética del cuidado de sí como práctica de la libertad", Obras esenciales, p. 1046.

${ }^{53}$ Foucault, Michel, Hermenéutica del sujeto, Fondo de Cultura Económica, Buenos Aires, 2014, p. 469. 
por los griegos -y recuperado por Foucault-, al modo cómo es comprendido en el cristianismo: amor hacia uno/a mismo/a, que se opone al cuidado de los/as otro/as. Por el contrario, para los griegos "no se trata de anteponer el cuidado de los otros al cuidado de sí; el cuidado de sí es éticamente lo primordial, en la medida en que la relación consigo mismo es ontológicamente la primera". ${ }^{54}$

En la Hermenéutica del sujeto, Foucault se ocupa de desarrollar el imperativo "cuídate de ti mismo" o "inquietud de sî" -epimeleia beautou-como una práctica reflexiva. 55 "Epimeleia beautou es la inquietud de sí mismo, el hecho de ocuparse de sí mismo, preocuparse de sí mismo". ${ }^{56}$ Esta noción presenta cuatro características: es una actitud -con respecto a sí mismo, a los otros y al mundo-, es una forma de atención - una mirada hacia uno mismo-, una serie de acciones o ejercicios -que se ejercen sobre uno mismo para transformarse-; $y$, por último, una manera de ser-una forma de reflexión, una actitud, unas prácticas. ${ }^{57}$ Estas prácticas supone una autotransformación del sujeto: el sujeto se (trans)forma a sí mismo por medio de ciertas prácticas. No se trata de una moral que lo constriñe a actuar de determinada manera, sino de un ejercicio que realiza el sujeto sobre sí mismo. En consecuencia, el cuidado de sí mismo es entendido como experiencia y también como técnica que elabora y transforma la experiencia del sujeto.

Esta consideración lo lleva a distinguir, e incluso oponer, espiritualidad de filosofía -entendida esta última en sentido tradicional. Por espiritualidad comprende la transformación que deber realizar el sujeto sobre sí mismo para acceder a la verdad. Por el contrario, la filosofía sería las condiciones a través

\footnotetext{
${ }^{54}$ Foucault, Michel, "La ética del cuidado de sí como práctica de la libertad", ed. cit., p. 1033.

55 Para Foucault estas prácticas de la relación del sujeto consigo mismo pasan a través del conocimiento de sí. Esto señala la relación entre sujeto y verdad. Pero la verdad ya no es la que objetiva al sujeto, sino que se encuentra ligada a su subjetividad. La verdad es una producción del sujeto, un acontecimiento subjetivo que pertenece a cada quien y no es el mismo para todos. Ver Chávez Muriel, Héctor R., Un acercamiento del concepto de sujeto en el pensamiento de Michel Foucault, Bogotá, Programa Editorial de la Universidad del Valle, 2012, p. 101.

${ }^{56}$ Foucault, Michel, Hermenéutica del sujeto, ed. cit., p. 17.

${ }^{57}$ Ibidem, pp. 28-29.
} 
de las cuales el sujeto puede ser capaz de verdad mediante el modelo de la práctica científica, el razonar rectamente y apelar a la evidencia. La filosofía no requiere de espiritualidad -de un ejercicio del sujeto sobre sí-, ya que es por la propia estructura del sujeto cognoscente que se puede alcanzar la verdad. Para Foucault la filosofía liquidó la espiritualidad, esto lo observa especialmente en Descartes y Kant. ${ }^{58}$ Como advierte Castro, "la lectura foucaultiana de la filosofía griega y romana tiende a mostrar, precisamente, cómo la espiritualidad formaba parte de la práctica filosófica". ${ }^{59}$

Foucault se ocupará de esa espiritualidad, de ese conjunto de prácticas, ejercicios y técnicas que dirigidas sobre sí mismo permiten al sujeto acceder a la verdad. Es en este sentido que podemos decir que la filosofía propuesta por Foucault es una filosofía de la práctica de sí, una filosofía que no debe comprenderse al modo en que Occidente caracterizó la filosofía moderna (como opuesta a la espiritualidad y meramente racional), sino más bien una filosofía que se liga a la espiritualidad, al trabajo que sobre sí realiza un sujeto y lo conduce hacia la verdad. Aquí podemos encontrar cierta similitud con Wittgenstein, quien también apela a la transformación del sujeto, sobre todo en el Tractatus, donde la espiritualidad aparece como práctica filosófica, como ejercicio de sí en el señalamiento de ese ámbito que llama lo místico. El ejercicio de sí que conduce a la verdad se muestra en tanto experiencia de rebasamiento de lo racional-discursivo.

Con la noción griega de parrhesía, Foucault establece una articulación entre actitud crítica y verdad, es una práctica de sí que liga al sujeto con la verdad. La parrbesía "debe comprenderse groseramente como franqueza, apertura de corazón, apertura de pensamiento, etc.”60 Aunque etimológicamente la parrhesía signifique "decirlo todo", en realidad se trata de un hablar con libertad, franqueza y apertura. ${ }^{61}$ Es una práctica de sí que implica una técnica -necesaria para transmitir el discurso veraz- pero, por eso mismo, un posicionamiento ético.

\footnotetext{
58 Ibidem, pp. 190-191.

${ }^{59}$ Castro, Edgardo, Introducción a Foucault, Buenos Aires, Siglo XXI, p. 133.

${ }^{60}$ Foucault, Michel, Hermenéutica del sujeto, p. 169.

${ }^{61}$ Ver Díaz, Esther, La filosofía de Michel Foucault, Buenos Aires, Biblos, 2014, p. 244.
} 
En El gobierno de síy de los otros define a la parrbesía como cierta manera de hablar, como una manera de decir la verdad. ${ }^{62}$ Es una manera de decir la verdad de modo tal que, por el hecho mismo de decirla, hay una exposición a un riesgo. Se trata de un riesgo ligado al decir veraz, que hace que quien habla francamente se convierta en interlocutor de sí mismo. "El parresiasta, quien utiliza la parrhesía, es el hombre verídico, esto es: quien tiene el coraje de arriesgar el decir veraz y que arriesga ese decir veraz en un pacto consigo mismo, en su carácter, justamente, de enunciador de verdad". ${ }^{63}$

La parrhesía va a ligar las nociones de sujeto, libertad y verdad de modo tal que se presenta como una forma en la cual el sujeto se liga a sí mismo a una verdad a través de un decir libre: "la parrhesía pone en juego una cuestión filosófica fundamental: nada menos que el lazo que se establece entre libertad y verdad". ${ }^{64}$ La parrhesía está vinculada al ethos, se trata de una cierta manera de ser y de comportarse. La parrbesía como arte de decir la verdad, como coraje de la verdad, ${ }^{65}$ es un hablar franco y sincero. El decir veraz que implica la parrhesía apunta a la formación de un ethos: una práctica de sí que hace que quien la ejercite ponga en juego su propia vida, ya que el decirlo todo expone a la muerte. Pero la filosofía, tal como es entendida tradicionalmente, no se pregunta por el riesgo que se asume en el decir con franqueza. Por el contrario, como expresa Gros, "la filosofía pregunta por la posibilidad general de la verdad, y ya no por el precio que debe pagar un sujeto por abrirse a la verdad". ${ }^{66}$

${ }^{62}$ Foucault logra articular el gobierno de sí y el gobierno de los otros por medio de la parrbesía, la cual presenta un sentido ético y político. La parrbesía puede comprenderse como un cierto modo de gobierno a través del decir veraz, un gobierno sobre uno mismo, en tanto que transforma al ethos, y un gobierno sobre los otros. Ver Torrano, Andrea, "Ética, Crítica y Estética en Michel Foucault. Un recorrido desde Kant hacia los griegos", en Rodríguez, N. B. y Viafara Sandoval, H. (comps.), Foucault, treinta años después. Aportes para pensar el problema del cuerpo y la educación, Cali, Editorial de la Universidad de La Plata y Universidad de San Buenaventura, 2016, pp. 51-75, y Castro, Edgardo, Introducción a Foucault, pp. 140-144.

${ }^{63}$ Foucault, Michel, El gobierno de síy de los otros, p. 82.

${ }^{64}$ Ibidem, p. 83.

${ }^{65} \mathrm{El}$ coraje por la verdad puede ser relacionado con el jsapere aude!, ten la audacia y el coraje de saber, lema de la Ilustración para Kant.

${ }^{66}$ Gros, Frédéric, Michel Foucault, Buenos Aires, Amorrortu, 2007, p. 145. 
Es esto último lo que quiere reavivar Foucault en el modo de hacer filosofía que propone.

En este sentido, en El coraje de la verdad, expresa que la parrhesía es una práctica, una práctica que tiene por correlato privilegiado, como punto de aplicación primordial, no la ciudad o el cuerpo de los ciudadanos a quienes debe convencerse, sino que se dirige y tiene efectos sobre la psykhé (el alma) del individuo. Por lo cual, el objetivo del decir veraz no es la salvación de la ciudad sino el ethos del individuo. Esto significa que la parrhesía "a la vez que se organiza en torno del principio del decir veraz, se encarna ahora en un conjunto de operaciones que permiten a la veridicción inducir en el alma efectos de transformación". ${ }^{67}$ La parrhesía es una práctica propiamente filosófica, se trata de una filosofía que debe concebirse como práctica de sí. Una práctica o ejercicio que liga al filósofo/a a la verdad, a una verdad que sólo puede enunciarse, decirse. De acuerdo con Revel, "he aquí, pues, el verdadero coraje de la verdad: el que consiste en desplazar los lugares del pensamiento y de lo político vaciándolos de sí mismos y reinvistiéndolos a partir de una práctica que sea, a la vez, interrogadora, crítica y no prescriptiva". 68

Respecto de este punto, lo que puede ser sabido y ser expresado, respecto de la relación del sujeto con la verdad, Wittgenstein y Foucault tomarán distintos senderos: en tanto el primero centra en los límites del lenguaje (para representar un hecho objetivo o para mostrar algo irrepresentable), la barrera entre el decir con sentido y el sinsentido; el segundo se afana en ese decir veraz, montando su estrategia en la exposición del sujeto del decir. En un caso se trata de guardar silencio, en el otro interesa (no guardarse de) decir la verdad.

\section{Conclusión}

${ }^{67}$ Foucault, Michel, El coraje de la verdad: El gobierno de síy de los otros II, Buenos Aires, Fondo de Cultura Económica, 2011, p. 82.

${ }^{68}$ Revel, Judith, Foucault, un pensamiento de lo discontinuo, Buenos Aires, Amorrortu, 2014, p. 222. 
Hemos tramado un recorrido por algunos de los lugares de contacto entre Wittgenstein y Foucault respecto al modo en que comprendieron la actividad filosófica, específicamente en torno a la noción de crítica y la práctica de sí. Los puntos de encuentro respecto de los caracteres de la práctica filosófica, lejos de permitirnos homogeneizar sus aportes y perspectivas, nos llevan a considerar por momentos puntos de cercanía y otros de cierta distancia en el modo en que emprenden dicha práctica, sin descontar que la crítica a la que se abocan no sólo señala hacia los límites del saber, sino también hacia el sentido teórico del mismo. Desde aquí, ambos señalan una apertura de la práctica crítica filosófica, en la que se juega también una crítica (a los límites) del sujeto y su relación con la verdad.

Quizá podría hacerse una objeción en torno a esta preocupación que observamos tanto en Wittgenstein como en Foucault sobre la actitud crítica y la práctica de sí -considerada como un ethos de quien emprenda la tarea filosófica- y la distinción que hicimos inicialmente entre la filosofía del sujeto y la filosofía del concepto, en la cual inscribíamos a Foucault y Wittgenstein. ${ }^{69}$ Aquí debemos aclarar que si bien ambos autores se han ocupado del sujeto que se transforma a sí mismo en la búsqueda de un saber o en su relación con la verdad, esto no debe conducirnos a pensar que se trata de una problemática que puede ser asimilada a la filosofía del sujeto. Por el contrario, esta preocupación en torno al sujeto y su relación con la filosofía como una práctica es una cuestión que debe inscribirse en la filosofía del concepto, en tanto se trata de un sujeto que es constituido por las normas del lenguaje (o reglas de lenguaje) y el poder, que se aleja de una concepción fenomenológica del sujeto, de una filosofía de la conciencia. En este sentido, huelga decir que es por y en un trabajo sobre los conceptos, sobre lo trascendental del lenguaje (tanto sus límites como condiciones de posibilidad) y sus potencialidades que el sujeto es puesto en cuestión, puesto en relación con la verdad y con la red de prácticas que lo configura. Acaso valga señalar que el alcance crítico de esta perspectiva es de suma relevancia en las dimensiones ética y política por las resonancias que genera, resonancias que no siempre se toman en cuenta y que hemos intentado aportar a lo largo de este trabajo.

69 Queremos agradecer especialmente a Pedro Karczmarczyk por esta observación y su atenta lectura. 
Pudimos advertir que en ambos pensadores se da cierta insatisfacción respecto al saber, en el reconocimiento de sus límites, pero a su vez, su crítica permite esclarecer el momento presente, actual y su potencialidad respecto del saber. Sobre este punto vale señalar que el rol de la filosofía es, o bien el de disolver los falsos problemas a partir del análisis del lenguaje circunscribiendo aquello sobre lo que es posible decir algo con sentido (Wittgenstein), o bien el de poner en cuestión los límites de saber en la construcción de una ontología de nosotros mismos (Foucault).

Las cercanías y distancias que encontramos entre los planteos de estos dos pensadores señalan un modo común de concebir la filosofía, no como un saber privilegiado por sobre otros, no como un conocimiento teórico que alude a una verdad en sentido mera y puramente epistémico, sino su apertura a una ética de la verdad. Este ethos compromete una práctica que supone tanto la crítica de lo dado como su transformación: un hacer, un ejercicio, una puesta en práctica que da como resultado una actitud, un modo de ser que vuelve sobre el sujeto.

La crítica radical que tanto Wittgenstein como Foucault ponen en marcha está ligada a considerar la tarea de la filosofía desde otra perspectiva: por un lado, ésta permite cartografiar (exteriormente) el conjunto de relaciones de cosas y sus usos concretos, las prácticas que involucran, las reglas que instauran. Por otro lado, transformar (internamente) al sujeto de ese saber crítico, indicando la potencia de la actitud que allí se pone en acto en relación con el modo de vida de aquél que la práctica.

En ambos, se daría cierta vuelta a la filosofía que no es sólo un retorno, sino volverla otra, apuntando a la fuerza viva que toca el ser mismo del sujeto. En Wittgenstein, se trata de una sabiduría que apunta a su transformación, donde la filosofía aparece como un ejercicio de sí, que supone un trabajo sobre sí mismo; en Foucault, se trata de volver a pensar la filosofía en relación con la espiritualidad que antaño era capaz de transformar al sujeto en relación a la verdad. En ambos, sin embargo, esta vuelta $a$ y de la filosofía se realiza -esto es central- bajo la premisa de ir más allá de las pretensiones de la metafísica.

Si bien cada uno traza modos diversos de realizar dicha actividad, el viraje respecto de la tradición filosófica es contundente en ambos: el giro que cada uno emprende está atravesado por un cambio de eje fundamental en el 
que frente a la teoría se acentúa la práctica, es decir, el sentido ético de la relación con el saber. Este cambio, huelga decirlo, no va en desmedro del esclarecimiento del lenguaje, ni de la clarificación de conceptos, ni de su contextualización o historización, más bien es por esos ejercicios que la filosofía puede considerarse como una práctica crítica y una práctica de sí.

Tanto en Wittgenstein como en Foucault, la filosofía es un trabajo sobre sí mismo que, lejos de ser pasivo, sostiene un pólemos: tanto en la crítica como en la práctica de sí. La crítica busca esclarecer, pero se da como una suerte de combate contra los encantamientos teóricos, contra las sedimentaciones del pensamiento, incluso contra los límites del lenguaje que nos constituyen y las prácticas que nos hacen. En una suerte de batalla contra la tradición filosófica, la crítica se erige como una actitud de transformación y puesta en cuestión de lo que comúnmente permanece incuestionado: sean éstos los límites de nuestro lenguaje, de nuestros saberes, la matriz histórica de los mismos. No se trata de encontrar esencias o el arché misterioso que sostiene la verdad, sino de clarificar, criticar, establecer la exterioridad de las redes (de proposiciones o de poderes) que sostienen tales verdades.

La filosofía es una práctica crítica del saber que hace eje en su matriz ética (no ya epistemológica), de lo que se trata no es de saber una verdad que deja intocado al sujeto de la filosofía, sino de la puesta en práctica de una verdad del ethos que lo transforma. La filosofía toca al sujeto, es una práctica de sí que involucra la asunción ética de la contradicción, de lo que rebasa nuestro saber y pensamiento, de una experiencia de lo irrepresentable (Wittgenstein), tanto como el coraje, la transformación, la mirada sobre sí (Foucault).

Si se parte de que la filosofía en Wittgenstein (en el Tractatus) es una suerte de terapéutica, si se considera que la búsqueda del último Foucault está atravesada por la inquietud de sí mismo, la intención que reúne a ambos es el modo en que la filosofía y la verdad se inscriben, incrustan y (trans)forman la vida, con-formando un modo de vida a través de una práctica de sí. Más allá de toda interpretación intelectualista, la causa de la búsqueda filosófica son aquellos problemas vitales, sobre los que poco o nada sabemos, pero que debemos tener el coraje de afrontar. La tesis 11 sobre Feuerbach ("Los filósofos no han hecho más que interpretar de diversos modos el mundo, pero de lo que se trata es de 
transformarlo"), tal como intuyó Shaviro, ${ }^{70}$ encuentra en estos filósofos una modulación tan vital como política: la consideración del ethos filosófico, implica tanto la crítica como transformación del mundo y de sí mismo/a.

70 Shaviro, Steven, "From language to forms of life. Theory and Practice in Wittgenstein", p. 216. 